\title{
A novel predictive model using routinely clinical parameters to predict liver fibrosis in patients with chronic hepatitis B
}

\author{
Jian Wang ${ }^{1}$, Xiaomin Yan ${ }^{2}$, Yue Yang ${ }^{1}$, Haiyan Chang ${ }^{1}$, Bei Jia ${ }^{2}$, Xiang-An Zhao ${ }^{3}$, \\ Guangmei Chen ${ }^{4}$, Juan Xia ${ }^{2}$, Yong Liư ${ }^{5}$, Yuxin Chen ${ }^{5}$, Guiyang Wang ${ }^{2}$, Li Wang ${ }^{2}$, \\ Zhaoping Zhang', Weimao Ding ${ }^{6}$, Rui Huang ${ }^{2}$ and Chao Wu ${ }^{1,2}$ \\ ${ }^{1}$ Department of Infectious Diseases, Nanjing Drum Tower Hospital, Clinical College of Nanjing Medical University, Nanjing, \\ Jiangsu, China \\ ${ }^{2}$ Department of Infectious Diseases, Nanjing Drum Tower Hospital, Nanjing University Medical School, Nanjing, Jiangsu, \\ China \\ ${ }^{3}$ Department of Infectious Diseases, Nanjing Drum Tower Hospital, Clinical College of Traditional Chinese and Western \\ Medicine, Nanjing University of Chinese Medicine, Nanjing, Jiangsu, China \\ ${ }^{4}$ Department of Infectious Diseases, Affiliated Hospital of Nanjing, University of Traditional Chinese Medicine, Nanjing, \\ Jiangsu, China \\ ${ }^{5}$ Department of Laboratory Medicine, Nanjing Drum Tower Hospital, Nanjing University Medical School, Nanjing, Jiangsu, \\ China \\ ${ }^{6}$ Department of Hepatology, Huai'an No. 4 People's Hospital, Huai'an, Jiangsu, China \\ Correspondence to: Chao Wu, email: dr.wu@nju.edu.cn
}

Rui Huang, email: doctor_hr@126.com

Keywords: chronic hepatitis B, liver biopsy, liver fibrosis, serum biomarkers, non-invasive fibrosis model Received: June 01, $2017 \quad$ Accepted: June 28, $2017 \quad$ Published: July 22, 2017

Copyright: Wang et al. This is an open-access article distributed under the terms of the Creative Commons Attribution License 3.0 (CC BY 3.0), which permits unrestricted use, distribution, and reproduction in any medium, provided the original author and source are credited.

\section{ABSTRACT}

Objectives: Noninvasive models have been established for the assessment of liver fibrosis in patients with chronic hepatitis B(CHB). However, the predictive performance of these established models remains inconclusive. We aimed to develop a novel predictive model for liver fibrosis in CHB based on routinely clinical parameters.

Results: Platelets(PLT), the standard deviation of red blood cell distribution width(RDW-SD), alkaline phosphatase(ALP) and globulin were independent predictors of significant fibrosis by multivariable analysis. Based on these parameters, a new predictive model namely APRG(ALP/PLT/RDW-SD/globulin) was proposed. The areas under the receiver-operating characteristic curves(AUROCs) of APRG index in predicting significant fibrosis( $\geq F 2$ ), advanced fibrosis( $\geq F 3$ ) and liver cirrhosis( $\geq F 4)$ were $0.757(95 \% \mathrm{CI} 0.699$ to $0.816), 0.763(95 \% \mathrm{CI} 0.711$ to 0.816$)$ and $0.781(95 \% \mathrm{CI} 0.728$ to 0.835$)$, respectively. The AUROCs of the APRG were significantly higher than that of aspartate transaminase(AST) to PLT ratio index(APRI), RDW to PLT ratio(RPR) and AST to alanine aminotransferase ratio(AAR) to predict significant fibrosis, advanced fibrosis and cirrhosis. The AUROCs of the APRG were also significantly higher than fibrosis-4 score (FIB-4) $(0.723,95 \%$ CI 0.663 to $\mathbf{0 . 7 8 3}$ ) for cirrhosis $(P=0.034)$ and better than gamma-glutamyl transpeptidase(GGT) to PLT ratio(GPR) $(0.657,95 \%$ CI 0.590 to 0.724$)$ for significant fibrosis $(P=0.001)$.

Materials and Methods: $308 \mathrm{CHB}$ patients who underwent liver biopsy were enrolled. The diagnostic values of the APRG for liver fibrosis with other noninvasive models were compared.

Conclusions: The APRG has a better diagnostic value than conventionally predictive models to assess liver fibrosis in CHB patients. The application of APRG may reduce the need for liver biopsy in CHB patients in clinical practice. 


\section{INTRODUCTION}

Hepatitis B virus (HBV) infection is a serious public health problem globally. Liver fibrosis and cirrhosis are major reasons of morbidity and mortality in patients with chronic hepatitis B (CHB) [1]. Assessing the stages of liver fibrosis in CHB patients could help clinicians predict the disease progression and formulate the optimally therapeutic schedule to avoid sever complications [2]. Therefore, it is essential to identify the degree of liver fibrosis to better manage such patients.

Currently, liver biopsy (LB) is recognized as the gold standard for estimating histological stages of liver diseases $[3,4]$. However, LB is an invasive and costly procedure which is very difficult to widespread utilization in routine practice. Meanwhile, sampling errors and observer discrepancy associated with liver biopsy may bias the result of liver fibrosis. Moreover, it does not allow the dynamic observation of liver fibrosis by LB. Therefore, non-invasive, inexpensive and convenient methods for assessing liver fibrosis are urgently needed.

Transient elastography (TE) is a promisingly noninvasive method that evaluates liver stiffness and has been reported to accurately assess the degree of liver fibrosis in CHB patients [5-7]. However, the technique is relatively high cost and susceptible by some factors such as necroinflammatory activity, total bilirubin (TBIL), obesity, which may also limit the clinical application $[8$, 9]. Simply noninvasive fibrosis tests (NITs) including aspartate transaminase (AST) to platelet (PLT) ratio index (APRI) and the fibrosis-4 score (FIB-4) using two or three inexpensive laboratory tests to predict hepatic fibrosis have been studied and validated over the past decade [1013]. WHO published the guideline on the management of CHB infection in 2015, which recommended the use of APRI as a noninvasive test to assess significant liver fibrosis and cirrhosis in resource-limited regions [8]. Both APRI and FIB-4 were based on patients with chronic hepatitis $\mathrm{C}(\mathrm{CHC})$ [11-13], while their value for assessing patients who are chronically infected with HBV remains controversial [14, 15]. Recently, Lemoine et al. reported the gamma-glutamyl transpeptidase (GGT) to PLT ratio (GPR) as a novel and more accurate laboratory marker than classical biomarkers APRI and FIB-4 to assess liver fibrosis in patients with CHB in West Africa populations, but this was not consistent in French cohort [16]. However, Schiavon et al. and Li et al. also demonstrated that GPR did not show better performance in a Brazilian cohort and a Chinese cohort $[17,18]$. Other serum-based models, such as AST to alanine aminotransferase (ALT) ratio (AAR), red cell distribution width (RDW) to PLT ratio (RPR) have also been reported to predict significant liver fibrosis and cirrhosis over the past decade in CHB patients, but they are somewhat difficult to use in clinical practice to perform the satisfactory outcomes [19-21]. Therefore, the aim of this study was to establish an improved model based on routinely clinical parameters for the assessment of liver fibrosis in treatment-naïve patients with CHB.

\section{RESULTS}

\section{Study population}

From January 2008 to December 2016, a total of 530 CHB patients who had undergone a liver biopsy were enrolled in this study. Overall, 70 patients were excluded base on exclusion criteria, 152 patients were excluded due to insufficient data. 308 patients who met the eligibility criteria were included as the final study cohort. Figure 1 presents the flow diagram of the study population. The median (and IQR) age of the CHB patients was 38.5 (29.0-47.0). 165 (53.6\%) CHB patients were positive for hepatitis B e antigen (HBeAg) and $230(74.7 \%)$ patients were male. The median (and IQR) ALT and AST level was $40.0(27.0,71.0) \mathrm{U} / \mathrm{L}$ and $34.0(25.0,50.0) \mathrm{U} / \mathrm{L}$, respectively. The distribution of each fibrosis stage in the subjects was as follows: F0, $32(10.4 \%)$ patients; F1, 53 (17.2\%) patients; F2, 47 (15.3\%) patients; F3, 74 (24.0\%) patients; and F4, 102 (33.1\%) patients. A detailed demographic and laboratory parameters of the subjects were shown in Table 1.

\section{Construction of a novel model for the assessment of liver fibrosis}

In the univariate analysis, the following parameters were identified as positively related to the significant fibrosis for the subjects: age $(\mathrm{P}<0.001)$, neutrophilic counts $(\mathrm{P}=0.024)$, red blood counts $(\mathrm{RBC})(\mathrm{P}=0.019)$, the standard deviation of the red blood cell distribution width (RDW-SD) $(\mathrm{P}<0.001)$, PLT $(\mathrm{P}<0.001)$, TBIL $(\mathrm{P}=0.045)$, globulin $(\mathrm{P}=0.001)$, alkaline phosphatase (ALP) $(\mathrm{P}=0.001)$, GGT $(\mathrm{P}=0.04)$, cholinesterase $(\mathrm{CHE})$ $(\mathrm{P}=0.008)$, international normalized ratio (INR) $(\mathrm{P}=0.019)$ and thrombin time (TT) $(\mathrm{P}=0.012)$. These significant parameters were selected for a multivariate analysis. In the multivariate analysis using the forward stepwise procedures, RDW-SD, PLT, globulin and ALP were the independent predictors of significant fibrosis (Table 2). Finally, using these independent predictors, a new model for predicting liver fibrosis, named the APRG (ALP/PLT/ RDW-SD/globulin) index, was derived as follows:

APRG index $=\frac{1}{1+e^{-(-6.091+A L P \times 0.015-P L T \times 0.01+R D W-S D \times 0.118+\text { globulin } \times 0.081)}}$

\section{Comparisons of different noninvasive models according to the fibrosis stages}

Figure 2 presents the levels of APRI, FIB-4, AAR, GPR, RPR and the APRG index in the CHB patients according to liver fibrosis stages. These NITs showed an 
increasing trend with fibrosis stages in CHB patients. The correlations between NITs and liver fibrosis stages were analyzed by the Spearman's rank correlation coefficient analysis. Fibrosis stages were positively correlated with APRI $(\mathrm{r}=0.363, \mathrm{P}<0.001)$, FIB-4 $(\mathrm{r}=0.447, \mathrm{P}<0.001)$, $\operatorname{AAR}(\mathrm{r}=0.143, \mathrm{P}=0.012)$ and $\operatorname{GPR}(\mathrm{r}=0.439, \mathrm{P}<0.001)$, RPR $(r=0.373, P<0.001)$ and the APRG index $(r=0.527$, $\mathrm{P}<0.001$ ) (Figure 3).

\section{Comparisons of AUROCs between the APRG index and other established NITs}

Predictive values of the six models were evaluated by the receiver operating characteristic (ROC) curves for the entire $\mathrm{CHB}$ population according to their histological fibrosis stages (Table 3 and Figure 4). The areas under the ROC curves (AUROCs) of the APRG index in predicting significant fibrosis $(\geq F 2)$, advanced fibrosis $(\geq F 3)$ and liver cirrhosis $(\geq \mathrm{F} 4)$ were 0.757 (95\%CI 0.699 to 0.816$)$, 0.763 (95\%CI 0.711 to 0.816$)$ and 0.781 (95\%CI 0.728 to 0.835$)$, respectively. The optimal cut-off values of the APRG index for predicting significant fibrosis, advanced fibrosis and cirrhosis were $0.695,0.777$ and 0.787 . For significant fibrosis, the AUROCs of the APRG index was significantly higher than APRI $(0.692,95 \% \mathrm{CI} 0.626$ to $0.758, \mathrm{P}=0.038)$, AAR $(0.616,95 \% \mathrm{CI} 0.549$ to 0.683 , $\mathrm{P}=0.002)$, GPR $(0.657,95 \%$ CI 0.590 to $0.724, \mathrm{P}=0.001)$ and RPR (0.681, 95\%CI 0.614 to $0.747, \mathrm{P}=0.006)$, while there were no significant differences between the AUROCs of the APRG index and FIB-4 (0.738, 95\%CI 0.678 to 0.799 , $\mathrm{P}=0.5$ ). For predicting advanced fibrosis, the AUROCs of the APRG index was significantly better than APRI $(0.689$, $95 \%$ CI 0.630 to $0.749, \mathrm{P}=0.012)$, AAR $(0.535,95 \%$ CI 0.470 to $0.600, \mathrm{P}<0.001)$ and RPR $(0.686,95 \% \mathrm{CI} 0.626$ to 0.746 , $\mathrm{P}=0.002)$, but was comparable with FIB-4 $(0.719,95 \% \mathrm{CI}$ 0.661 to $0.777, \mathrm{P}=0.093)$ and $\operatorname{GPR}(0.737,95 \% \mathrm{CI} 0.681$ to $0.794, \mathrm{P}=0.383$ ). For predicting cirrhosis, the APRG index exhibited a significantly higher AUROCs compared with APRI $(0.676,95 \%$ CI 0.612 to $0.740, \mathrm{P}<0.001)$, FIB$4(0.723,95 \%$ CI 0.663 to $0.783, \mathrm{P}=0.034)$, AAR (0.574, $95 \%$ CI 0.509 to $0.640, \mathrm{P}<0.001)$ and RPR $(0.709,95 \% \mathrm{CI}$ 0.647 to $0.771, \mathrm{P}=0.009$ ), while there were no significant differences between the AUROCs of the APRG index and GPR $(0.758,95 \%$ CI 0.702 to $0.814, \mathrm{P}=0.458)$.

\section{DISCUSSION}

Early detection and accurate assessment the severity of liver fibrosis is essential for antiviral therapy decisions in CHB patients [8]. Considering the limitations of LB, a simple predictive model for liver fibrosis using routinely serum-based biomarkers has always been an urgent assignment for clinician to avoid unnecessary LB. Although several non-invasive methods involving

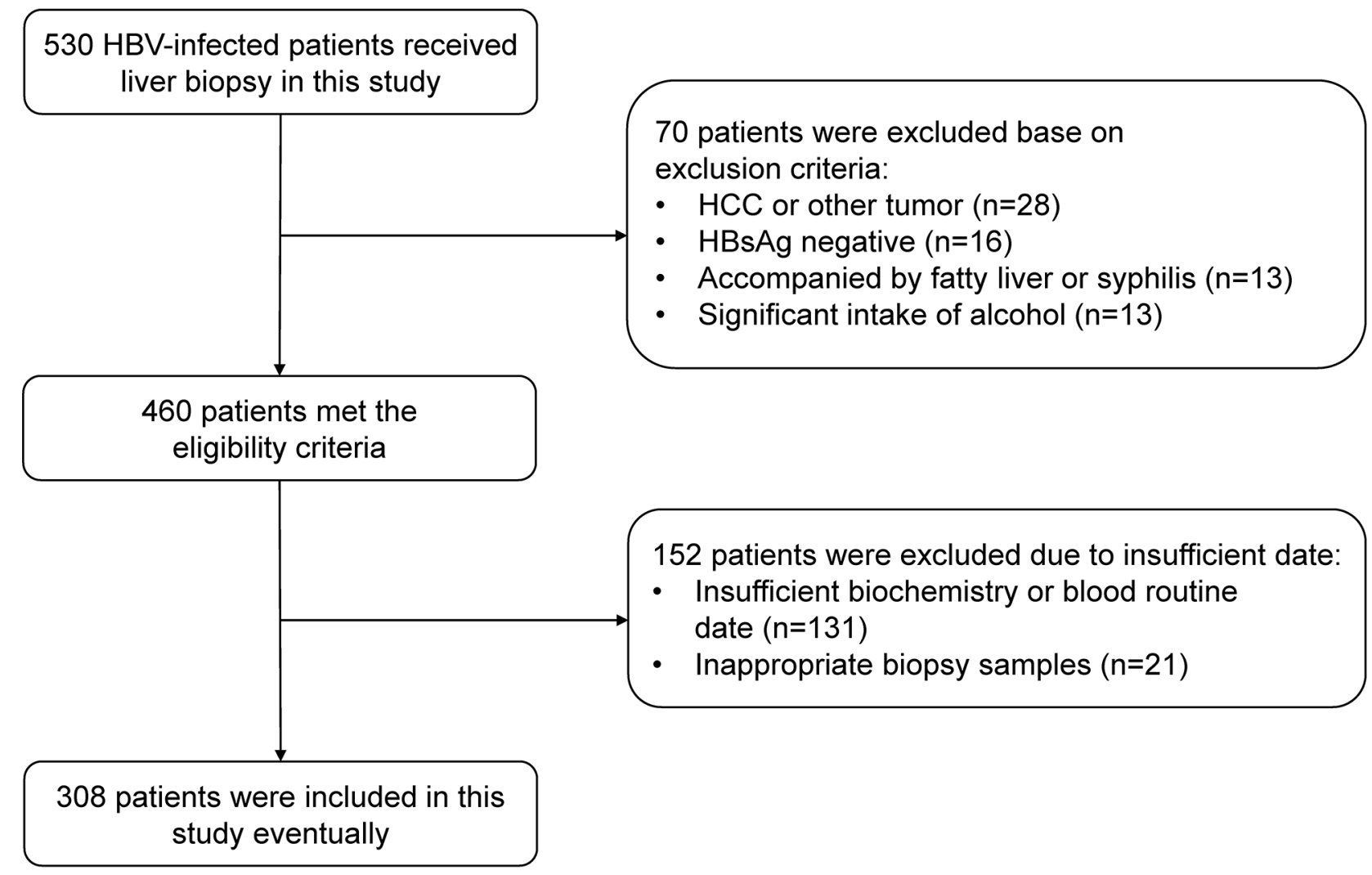

Figure 1: Flow diagram describing the selection of the study population. 
Table 1: Baseline characteristics of the subjects

\begin{tabular}{|c|c|}
\hline Characteristics & CHB $(n=308)$ \\
\hline Median Age (years) (IQR) & $38.5(29.0,47.0)$ \\
\hline Male $(\%)$ & $230(74.7)$ \\
\hline Median RBC $\left(10^{12} / \mathrm{L}\right)(\mathrm{IQR})$ & $4.6(4.2,5.0)$ \\
\hline Median Neutrophils (10/L) (IQR) & $2.8(2.1,3.5)$ \\
\hline Median Lymphocytes $\left(10^{9} / \mathrm{L}\right)(\mathrm{IQR})$ & $1.6(1.3,2.0)$ \\
\hline Median $\mathrm{Hb}(\mathrm{g} / \mathrm{L})$ (IQR) & $154.0(145.0,164.0)$ \\
\hline Median RDW-CV (\%) (IQR) & $12.6(12.0,13.5)$ \\
\hline Median RDW-SD (fL) (IQR) & $46.9(43.5,49.3)$ \\
\hline Median PLT $\left(10^{9} / \mathrm{L}\right)(\mathrm{IQR})$ & $158.0(118.0,195.8)$ \\
\hline Median TBIL (umol/L) (IQR) & $15.2(11.8,20.9)$ \\
\hline Median Albumin (g/L) (IQR) & $45.4(41.6,47.5)$ \\
\hline Median Globulin (g/L) (IQR) & $27.8(24.4,31.0)$ \\
\hline Median ALT (U/L) (IQR) & $40.0(27.0,71.0)$ \\
\hline Median AST (U/L) (IQR) & $34.0(25.0,50.0)$ \\
\hline Median ALP (U/L) (IQR) & $70.0(59.3,90.8)$ \\
\hline Median GGT (U/L) (IQR) & $33.0(21.0,65.8)$ \\
\hline Median LDH (U/L) (IQR) & $174.0(148.4,195.0)$ \\
\hline Median CHE (U/L) (IQR) & $7452.5(5846.3,8925.8)$ \\
\hline Median TT (s) (IQR) & $17.0(16.3,17.9)$ \\
\hline Median INR (IQR) & $1.2(1.1,1.2)$ \\
\hline $\mathrm{HBeAg}$ positive $(\%)$ & $165(53.6)$ \\
\hline \multicolumn{2}{|l|}{ Fibrosis stages } \\
\hline $\mathrm{F} 0(\%)$ & $32(10.4)$ \\
\hline $\mathrm{F} 1(\%)$ & $53(17.2)$ \\
\hline $\mathrm{F} 2(\%)$ & $47(15.3)$ \\
\hline $\mathrm{F} 3(\%)$ & $74(24.0)$ \\
\hline F4(\%) & $102(33.1)$ \\
\hline
\end{tabular}

RBC: red blood cell; $\mathrm{Hb}$ : hemoglobin; RDW-CV: the coefficient of variation of the red blood cell distribution width; RDW-SD: the standard deviation of the red blood cell distribution width; PLT: platelets; TBIL: total bilirubin; ALT: alanine aminotransferase; AST: aspartate aminotransferase; ALP: alkaline phosphatase; GGT: gamma-glutamyl transpeptidase; LDH: lactic dehydrogenase; CHE: cholinesterase; TT: thrombin time; INR: international normalized ratio; HBeAg: Hepatitis B e antigen.

blood biomarkers such as APRI and FIB-4 to predict liver fibrosis have been developed over the past decade $[13,26]$, these methods were based on patients with CHC and the accuracy for assessing patients with CHB remains controversial. Recently, several models such as GPR, RPR and AAR have been used to assess the liver fibrosis of patients with CHB, but the accuracy and reliability of these indexes are not very satisfactory in predicting liver fibrosis $[17,27,28]$.
In present study, we aimed to develop a novel inexpensive and routinely available model to predict significant fibrosis, advanced fibrosis and cirrhosis using blood-based parameters. Blood routine examination and biochemistry parameters, which are commonly tested during course of $\mathrm{CHB}$ patients, have essential implications for the natural history of chronic HBV infection. The PLT count is an independent risk factor in the most predictive models for liver fibrosis and cirrhosis. A large 
Table 2: Univariate and multivariate analyses of the relationships between hematological parameters and the significant fibrosis in the entire patients

\begin{tabular}{|c|c|c|c|c|}
\hline \multirow[t]{2}{*}{ Variables } & \multicolumn{4}{|c|}{ Significant fibrosis (F2-F4) } \\
\hline & Univariate & P-value & Multivariate & P-value \\
\hline Age & $1.047(1.022,1.073)$ & $<0.001$ & & \\
\hline Neutrophils & $0.795(0.651,0.970)$ & 0.024 & & \\
\hline Lymphocytes & $0.708(0.468,1.072)$ & 0.103 & & \\
\hline Monocytes & $0.421(0.056,3.182)$ & 0.402 & & \\
\hline $\mathrm{RBC}$ & $0.559(0.339,0.909)$ & 0.019 & & \\
\hline $\mathrm{Hb}$ & $0.999(0.986,1.012)$ & 0.832 & & \\
\hline RDW-CV & $1.014(0.851,1.208)$ & 0.877 & & \\
\hline RDW-SD & $1.135(1.063,1.212)$ & $<0.001$ & $1.125(1.048,1.207)$ & 0.001 \\
\hline PLT & $0.989(0.985,0.994)$ & $<0.001$ & $0.990(0.985,0.995)$ & $<0.001$ \\
\hline TBIL & $1.034(1.001,1.069)$ & 0.045 & & \\
\hline Albumin & $0.965(0.908,1.025)$ & 0.245 & & \\
\hline Globulin & $1.097(1.038,1.116)$ & 0.001 & $1.084(1.018,1.155)$ & 0.012 \\
\hline ALT & $1.001(0.999,1.004)$ & 0.303 & & \\
\hline AST & $1.005(0.999,1.012)$ & 0.119 & & \\
\hline ALP & $1.02(1.008,1.032)$ & 0.001 & $1.015(1.002,1.028)$ & 0.021 \\
\hline GGT & $1.005(1.000,1.009)$ & 0.04 & & \\
\hline LDH & $1.003(0.997,1.009)$ & 0.379 & & \\
\hline $\mathrm{CHE}$ & $1.000(1.000,1.000)$ & 0.008 & & \\
\hline INR & $\begin{array}{c}14.857(1.554 \\
142.066)\end{array}$ & 0.019 & & \\
\hline TT & $1.271(1.055,1.531)$ & 0.012 & & \\
\hline
\end{tabular}

RBC: red blood cell; Hb: hemoglobin; RDW-CV: the coefficient of variation of the red blood cell distribution width; RDW-SD: the standard deviation of the red blood cell distribution width; PLT: platelets; TBIL: total bilirubin; ALT: alanine aminotransferase; AST: aspartate aminotransferase; ALP: alkaline phosphatase; GGT: gamma-glutamyl transpeptidase; LDH: lactic dehydrogenase; CHE: cholinesterase; TT: thrombin time; INR: international normalized ratio.

amount of studies reported that low PLT counts were associated with advanced liver fibrosis [29-32]. A possible explanation may be that the decreased PLT counts are due to splenomegaly and the decreased thrombopoietin production associated with liver cell failure in cirrhosis patients $[33,34]$. RDW is a measure of the heterogeneity of the volume of red blood cells and is often used to diagnose different types of anemia. Recently, RDW has been demonstrated to be associated with cardiovascular and pulmonary diseases [35-37]. Felker et al. identified that high RDW was a strong independent predictor of outcome in patients with chronic heart failure [35]. Hampole et al. reported that RDW value was an independent predictor of mortality in patients with pulmonary hypertension [37]. RDW was also demonstrated as an independent predictor of the liver fibrosis stage in CHB patients. A retrospective study by Chen et al. showed that the RDW was a strong predictor of liver fibrosis in patients with CHB [28]. Lee et al. developed a novel index, the RPR, by using RDW and PLT. They found that RPR was comparable to APRI and FIB-4 but was inferior to TE for assessing significant fibrosis in a Korean population with chronic HBV infection [38]. However, it is worth noting that RDW can be expressed as RDW-SD and the coefficient of variation of the red blood cell distribution width (RDW-CV). Our findings indicated that RDW-CV was not a predictive risk factor for liver fibrosis. Instead, the RDW-SD was a strong and independent predictor of the liver fibrosis. $\mathrm{RDW}-\mathrm{CV}$ is calculated from the erythrocyte volume distribution histogram. It represents the coefficient of variation of erythrocyte volume around mean corpuscular volume (MCV), while RDW-SD is determined from the 
width of erythrocyte volume distribution curve at level $20 \%$ above baseline and is expressed in femtoliters [39]. Caporal et al. repotted RDW-CV had higher sensitivity and efficiency in detecting anisocytosis in microcytic $\mathrm{MCV}$ ranges compared with RDW-SD. However, in normocytic and macrocytic $\mathrm{MCV}$ ranges, RDW-SD showed better efficiency in detecting anisocytosis than
RDW-CV [40]. The reason why RDW-SD is associated with the stages of liver fibrosis is unclear and deserves further investigation. In addition, Schmilovitz-Weiss et al. and $\mathrm{Xu}$ et al. reported that there appears to be a strong association between serum level of globulin and extent of liver fibrosis in patients with chronic HBV infection [41, 42]. The correlation between globulin level
A

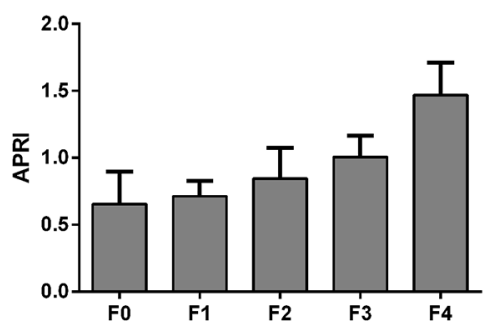

D

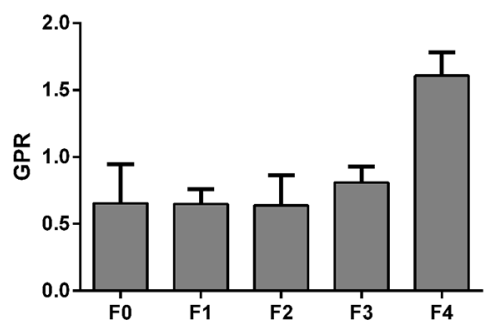

B

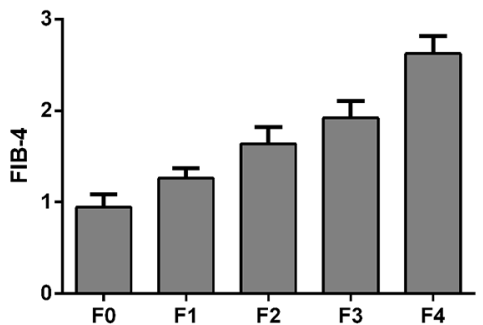

E

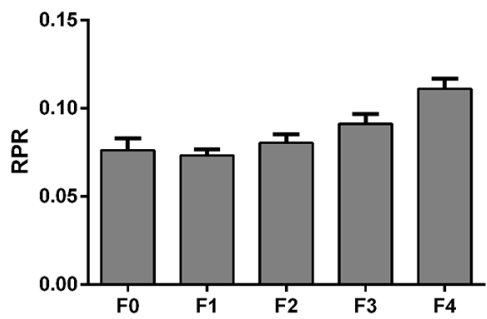

C

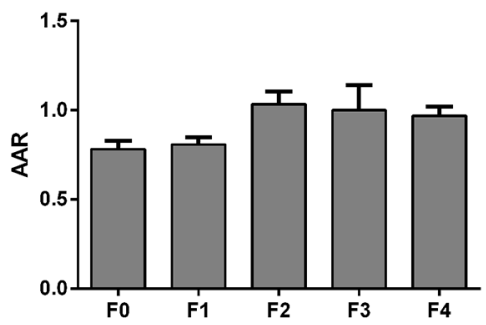

$\mathbf{F}$

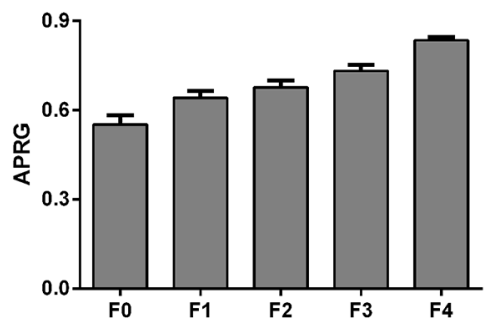

Figure 2: Comparisons of different noninvasive models according to the liver fibrosis stages in CHB patients.

A

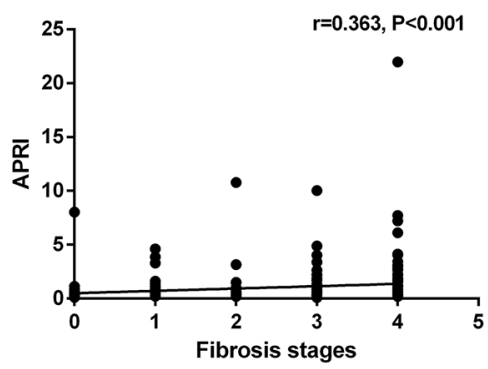

D

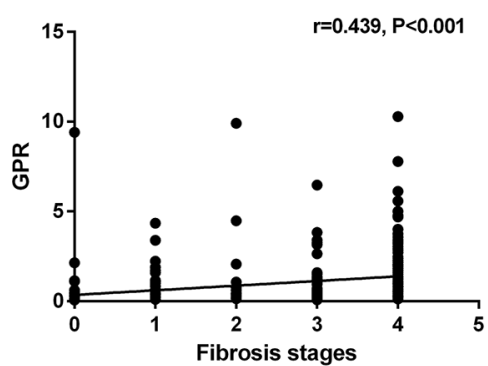

B

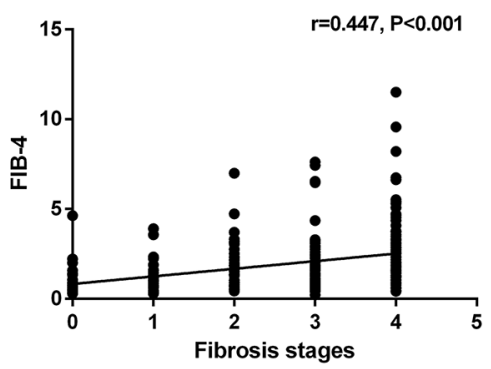

E

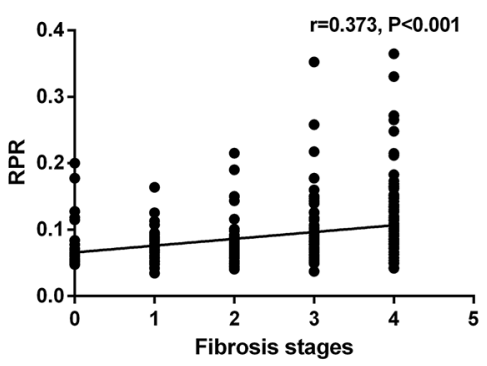

C

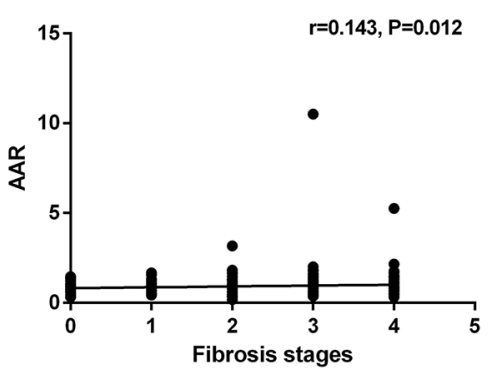

F

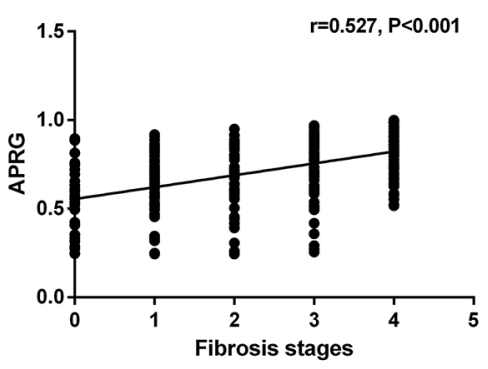

Figure 3: Correlations between different noninvasive models and liver fibrosis stages. 
Table 3: Diagnostic accuracy of different indexes for the prediction of liver fibrosis in the CHB patients

\begin{tabular}{ccccccc}
$\begin{array}{c}\text { Optimized } \\
\text { cutoff }\end{array}$ & $\begin{array}{c}\text { Sensitivity } \\
(\%)\end{array}$ & $\begin{array}{c}\text { Specificity } \\
(\%)\end{array}$ & AUC (95\%CI) & LR + LR - P value & $\begin{array}{c}\text { P value } \\
\text { of ROC } \\
\text { contrast test* }\end{array}$ \\
\hline
\end{tabular}

\section{F0-F1 vs. F2-F4}

\begin{tabular}{|c|c|c|c|c|c|c|c|c|}
\hline APRI & 0.544 & 65.47 & 70.59 & $0.692(0.626,0.758)$ & 2.226 & 0.489 & $<0.001$ & 0.038 \\
\hline FIB-4 & 1.205 & 68.16 & 71.76 & $0.738(0.678,0.799)$ & 2.414 & 0.444 & $<0.001$ & 0.500 \\
\hline AAR & 0.802 & 60.54 & 63.53 & $0.616(0.549,0.683)$ & 1.660 & 0.621 & 0.002 & 0.002 \\
\hline GPR & 0.432 & 60.09 & 70.59 & $0.657(0.590,0.724)$ & 2.043 & 0.565 & $<0.001$ & 0.001 \\
\hline RPR & 0.070 & 70.85 & 63.53 & $0.681(0.614,0.747)$ & 1.943 & 0.459 & $<0.001$ & 0.006 \\
\hline APRG & 0.695 & 73.54 & 68.24 & $0.757(0.699,0.816)$ & 2.316 & 0.388 & $<0.001$ & - \\
\hline \multicolumn{9}{|c|}{ F0-F2 vs. F3-F4 } \\
\hline APRI & 0.667 & 58.52 & 75.00 & $0.689(0.630,0.749)$ & 2.341 & 0.553 & $<0.001$ & 0.012 \\
\hline FIB-4 & 1.086 & 77.84 & 59.09 & $0.719(0.661,0.777)$ & 1.903 & 0.375 & $<0.001$ & 0.093 \\
\hline AAR & 0.917 & 42.61 & 67.42 & $0.535(0.470,0.600)$ & 1.308 & 0.851 & 0.295 & $<0.001$ \\
\hline GPR & 0.413 & 71.59 & 69.70 & $0.737(0.681,0.794)$ & 2.363 & 0.408 & $<0.001$ & 0.383 \\
\hline RPR & 0.090 & 48.30 & 82.58 & $0.686(0.626,0.746)$ & 2.773 & 0.626 & $<0.001$ & 0.002 \\
\hline APRG & 0.777 & 63.64 & 78.03 & $0.763(0.711,0.816)$ & 2.897 & 0.466 & $<0.001$ & - \\
\hline \multicolumn{9}{|c|}{ F0-F3 vs. F4 } \\
\hline APRI & 0.667 & 64.71 & 66.02 & $0.676(0.612,0.740)$ & 1.904 & 0.535 & $<0.001$ & $<0.001$ \\
\hline FIB-4 & 1.602 & 65.69 & 71.84 & $0.723(0.663,0.783)$ & 2.333 & 0.478 & $<0.001$ & 0.034 \\
\hline AAR & 0.767 & 73.53 & 45.15 & $0.574(0.509,0.640)$ & 1.341 & 0.586 & 0.034 & $<0.001$ \\
\hline GPR & 0.482 & 74.51 & 67.96 & $0.758(0.702,0.814)$ & 2.326 & 0.375 & $<0.001$ & 0.458 \\
\hline RPR & 0.070 & 85.29 & 50.49 & $0.709(0.647,0.771)$ & 1.723 & 0.291 & $<0.001$ & 0.009 \\
\hline APRG & 0.787 & 70.59 & 71.36 & $0.781(0.728,0.835)$ & 2.465 & 0.412 & $<0.001$ & - \\
\hline
\end{tabular}

AUROC: area under the receiver operating characteristic curve; CI: confidence interval; LR-: negative likelihood ratio; LR+: positive likelihood ratio; AAR: aspartate aminotransferase to alanine aminotransferase ratio; APRI: aspartate aminotransferase to platelet ratio index; FIB-4: fibrosis index based on the four factors; GPR: gamma-glutamyl transpeptidase to platelet ratio; RPR: red cell distribution width to platelet ratio.
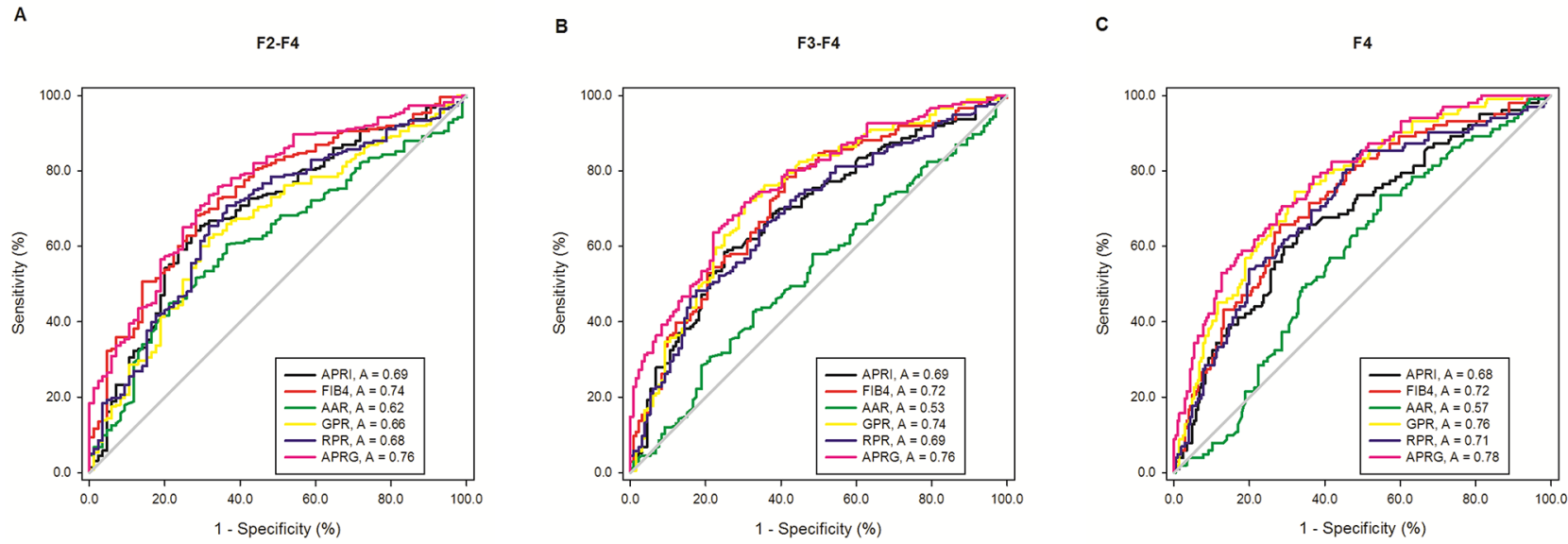

Figure 4: Receiver operating characteristic (ROC) curves of noninvasive blood biomarkers, including APRI, FIB-4, GPR, RPR, AAR and the APRG index level for significant liver fibrosis (A) advanced liver fibrosis (B) and liver cirrhosis (C) in the CHB patients. 
and the degree of liver fibrosis was also found in our study through multivariate regression analysis. ALP is a hydrolase enzyme which is mainly expresses in the liver, bile duct, bone and so on [43]. In previous study, ALP has been also identified to be an independent predictor to liver fibrosis in CHB patients [44]. We found by forward stepwise multiple regression analysis, that ALP was also significantly correlated with different fibrosis stages. In the present study, other variables such as GGT, TBIL, AST, thrombin time (TT) and age were not the predictive factors for liver fibrosis. Conflicting findings are reported in previous studies on these serum parameters being independent variables determining the high fibrosis scores $[16,23,30]$. These discrepancies may due to the different study cohorts, which had liver disease caused by different pathogenic factors. Eventually, through multivariate analysis, RDW-SD, PLT, globulin and ALP were identified as the independent predictors of significant fibrosis in our study. Therefore, the APRG index was developed based on these four independent predictors of significant fibrosis.

The APRG index obtained higher AUCs than the APRI, FIB-4, RPR, GPR, AAR in the prediction of liver fibrosis. The APRG index is an accurately predictive index of significant fibrosis, advanced fibrosis and cirrhosis as compared with the APRI. Although WHO guideline suggests the use of APRI for estimating liver fibrosis in patients with $\mathrm{CHB}$ [8], many studies demonstrated that APRI and FIB-4 could identify liver fibrosis with an only moderate sensitivity and accuracy in CHB patients, and were not an ideal replacement for liver biopsy [14, $44,45]$. FIB-4 was first reported by Sterling et al. and was demonstrated to be useful for the diagnosis of liver fibrosis in CHC patients with co-infected HIV [23]. However, the diagnostic value of FIB-4 is still limited and controversial for chronically $\mathrm{HBV}$-infected patients $[16,21]$. In our study, although the diagnostic value of the APRG index was comparable with FIB-4 in identifying significant fibrosis and advanced fibrosis in CHB patients, the performance of the APRG index was slightly superior to that of FIB-4 in distinguishing liver cirrhosis. GPR is a newly noninvasive index to assess liver fibrosis in chronically HBV-infected patients and is superior to APRI and FIB-4 in West Africa cohorts [16]. However, Li et al. and Schiavon LL et al. found that GPR did not show any advantage in a Chinese cohort and a Brazilian cohort [17, 18]. In the present study, we found that as compared with the GPR, the APRG index had a more powerful predictive value for significant fibrosis but had equally powerful in the prediction of advanced fibrosis and cirrhosis. We assumed that the discrepancies in the diagnostic accuracy of GPR may be caused by laboratory methods, HBV genotypes and demographic characteristics. Although the GPR is a promising index for predicting liver fibrosis and includes only two inexpensive serum parameters, the value of GPR for distinguishing liver fibrosis in patients with $\mathrm{CHB}$ needs to be determined in further studies. Chen et al. reported that RPR is an accurately predictive index in identify significant fibrosis compared to APRI and FIB-4 [28]. However, Lee et al. verified that RPR has a similar performance with APRI and FIB-4 for assessing significant fibrosis in a Korean cohort of CHB [38]. Our study shown that RPR was inferior to the APRG index in identifying significant fibrosis, advanced fibrosis and cirrhosis. Other models, such as AAR was also proposed to assess the stages of liver fibrosis in patients with $\mathrm{CHB}$ recent years [27]. However, our study suggests that AAR is not a good method for the estimation of fibrosis stage compared with the APRG index.

The present study has several limitations. Firstly, our study is a retrospective study and the data was obtained from a single center. Secondly, our study did not consider the impact of the HBV genotypes, although the HBV genotypes of Asian are usually B or C [46]. Thirdly, since the parameters of serum HBV marker was insufficient, we did not consider the impact of these markers in liver fibrosis, and these factors should be considered in future studies. Fourthly, we did not compare the performance of APRG with TE for diagnosing liver fibrosis in CHB patients due to the absence of available data. Thus, whether the APRG is superior to TE for diagnosing liver fibrosis in CHB patients deserves further investigation.

\section{MATERIALS AND METHODS}

\section{Patients}

The present study included treatment-naïve patients who underwent LB at Huai'an No. 4 People's Hospital (Jiangsu, China) from 2008 to 2016 . CHB is defined as having blood that tested positive for serum HBV surface antigen $(\mathrm{HBsAg})>6$ months. Patients were excluded from this study for the following reasons: co-infection with hepatitis $\mathrm{C}$ virus (HCV), hepatitis $\mathrm{D}$ virus (HDV) and human immunodeficiency virus (HIV), primary biliary cirrhosis, autoimmune hepatitis, Wilson's disease, hepatocellular carcinoma (HCC) or other types of cancer, hematological diseases, acute heart failure and pregnancy.

All subjects provided written informed consent for the liver biopsy. The Ethics Committee of the Huai'an No. 4 People's Hospital, Jiangsu, China, approved the consent procedure and the study.

\section{Liver biopsy and laboratory test}

LB was performed using 16-gauge biopsy needles under ultrasound guidance. To be considered as adequate for scoring, a minimum of $1 \mathrm{~cm}$ of liver tissue with at least five portal tracts was required. All the LB specimens were processed by formalin fixation, paraffin-embedding and stained with hematoxylin and eosin. All histological specimens were reviewed by pathologist blinded to patient clinical characteristics, according to the METAVIR 
scoring system [22]. Liver fibrosis was classified into the following five stages: F0, no fibrosis; F1, portal fibrosis without septa; F2, portal fibrosis with rare septa; F3, numerous septa without cirrhosis; and F4, cirrhosis [22]. Patient demographic and laboratory parameters were collected at the time of liver biopsy.

\section{Noninvasive prediction methods and calculation formulae}

The noninvasive prediction methods and calculation formulae used in our study were as follows: APRI: (AST $(\mathrm{U} / \mathrm{L}) / \mathrm{ULN}$ of AST)/PLT count $\left(10^{9} / \mathrm{L}\right) \times 100$ [11]; FIB4: $($ age $($ years $) \times$ AST $(\mathrm{U} / \mathrm{L})) /\left(\left(\mathrm{PLT}\right.\right.$ count $\left(10^{9} / \mathrm{L}\right) \times(\mathrm{ALT}$ $\left.(\mathrm{U} / \mathrm{L}))^{1 / 2}\right)$ [23]; AAR: AST (U/L)/ALT (U/L) [24]; GPR: $\left(\mathrm{GGT}(\mathrm{U} / \mathrm{L}) / \mathrm{ULN}\right.$ of GGT)/PLT count $\left(10^{\%} / \mathrm{L}\right) \times 100$ [16]; RPR: RDW (\%)/PLT count $\left(10^{9} / \mathrm{L}\right)$ [25].

\section{Statistical analyses}

The data analysis was performed using SPSS version 22.0 software (SPSS Inc., Chicago, IL, United States) and SigmaPlot version 12.5 (Systat Software Inc., San Jose, CA, United States). Continuous variables are expressed as median (interquartile range) and were compared using the Mann-Whitney U test. Categorical data were reported as percentages. Correlations were evaluated by the Spearman's rank correlation coefficient analysis. The risk factors for liver fibrosis in patients with $\mathrm{CHB}$ were analyzed by binary logistic regression. Variables having $P$ values $<0.05$ in the univariate analysis were used for a multivariate stepwise logistic regression analysis. Binary logistic regression analyses were used to develop the predictive models of liver fibrosis and the final prediction model was selected using the forward stepwise procedures. Probabilities generated from the predictive models were recorded and used as new input variables for the ROC curve analysis. The diagnostic accuracy of serum model for liver fibrosis was evaluated by using the ROC curve. The AUROCs as well as 95\% confidential interval (CI) of AUROC were calculated. Differences between the AUROCs were tested using the z-test. The cut-off values were determined by the Youden index which was the optimal combination of sensitivity and specificity. All $\mathrm{P}$-value were 2 -sided and any value of $\mathrm{P}<0.05$ was considered statistically significant.

\section{CONCLUSIONS}

In summary, the present study demonstrated that PLT, RDW-SD, ALP and globulin were independent variables for determining the severity of liver fibrosis. The APRG index that is established using these four parameters has a better diagnosis performance compared to conventional blood biomarkers, such as APRI, FIB-4, GPR, RPR and AAR. Multi-center, prospective cohort studies are needed to confirm the diagnostic values of the APRG index and compare it with other established NITs for $\mathrm{CHB}$ patients in the future.

\section{Abbreviations}

CHB: chronic hepatitis B; HBV: hepatitis B virus; $\mathrm{CHC}$ : chronic hepatitis $\mathrm{C}$; $\mathrm{HCV}$ : hepatitis $\mathrm{C}$ virus; HDV: hepatitis D virus; HIV: human immunodeficiency virus; HCC: hepatocellular carcinoma; HBsAg: hepatitis B surface antigen; HBeAg: hepatitis B e antigen; LB: liver biopsy; TE: transient elastography; RBC: red blood cell; $\mathrm{Hb}$ : hemoglobin; RDW-CV: the coefficient of variation of the red blood cell distribution width; RDW-SD: the standard deviation of the red blood cell distribution width; PLT: platelets; MCV: mean corpuscular volume; TBIL: total bilirubin; ALT: alanine aminotransferase; AST: aspartate aminotransferase; ALP: alkaline phosphatase; GGT: gamma-glutamyl transpeptidase; LDH: lactic dehydrogenase; CHE: cholinesterase; TT: thrombin time; INR: international normalized ratio; AUROC: area under the receiver operating characteristic curve; CI: confidence interval; LR-: negative likelihood ratio; $\mathrm{LR}+$ : positive likelihood ratio; AAR: aspartate aminotransferase to alanine aminotransferase ratio; APRI: aspartate aminotransferase to platelet ratio index; FIB-4: fibrosis index based on the four factors; GPR: gamma-glutamyl transpeptidase to platelet ratio; RPR: red cell distribution width to platelet ratio; NITs: noninvasive fibrosis tests.

\section{Author contributions}

Study concept and design: Rui Huang, Chao Wu and Jian Wang; analysis and interpretation of the data: Yuxin Chen, Juan Xia, Xiaomin Yan, Yong Liu, Li Wang, Bei Jia and Guiyang Wang; collection of data: Guangmei Chen, Zhaoping Zhang, Xiang-An Zhao, Weimao Ding, Yue Yang and Haiyan Chang; drafting the manuscript: Jian Wang, Rui Huang and Chao Wu.

\section{CONFLICTS OF INTEREST}

The authors have declared that no conflicts of interest exist.

\section{FUNDING}

The study was supported from the National Natural Science Foundation of China (81672025), Medical Science and Technology Development Foundation of Nanjing (ZDX16004 and YKK16118) and Natural Science Foundation of Jiangsu Province for Young Scholar (BK20160121). Nanjing Science and Technology Development Plan (201503016), Jiangsu Provincial Medical Innovation Team (CXTDA2017005). 


\section{REFERENCES}

1. Lok AS, McMahon BJ. Chronic hepatitis B: update 2009. Hepatology. 2009; 50: 661-662.

2. Xu XY, Kong H, Song RX, Zhai YH, Wu XF, Ai WS, Liu HB. The effectiveness of noninvasive biomarkers to predict hepatitis B-related significant fibrosis and cirrhosis: a systematic review and meta-analysis of diagnostic test accuracy. PLoS One. 2014; 9.

3. Terrault NA, Bzowej NH, Chang KM, Hwang JP, Jonas MM, Murad MH, American Association for the Study of Liver Diseases. AASLD guidelines for treatment of chronic hepatitis B. Hepatology. 2016; 63: 261-283.

4. Sarin SK, Kumar M, Lau GK, Abbas Z, Chan HL, Chen CJ, Chen DS, Chen HL, Chen PJ, Chien RN, Dokmeci AK, Gane E, Hou JL, et al. Asian-Pacific clinical practice guidelines on the management of hepatitis B: a 2015 update. Hepatol Int. 2016; 10: 1-98.

5. Seo YS, Kim MY, Kim SU, Hyun BS, Jang JY, Lee JW, Lee JI, Suh SJ, Park SY, Park H, Jung EU, Kim BS, Kim $\mathrm{IH}$, et al. Accuracy of transient elastography in assessing liver fibrosis in chronic viral hepatitis: a multicentre, retrospective study. Liver Int. 2015; 35: 2246-2255.

6. Degos F, Perez P, Roche B, Mahmoudi A, Asselineau J, Voitot H, Bedossa P, FIBROSTIC study group. Diagnostic accuracy of FibroScan and comparison to liver fibrosis biomarkers in chronic viral hepatitis: a multicenter prospective study (the FIBROSTIC study). J Hepatol. 2010; 53: 1013-1021.

7. Marcellin P, Ziol M, Bedossa P, Douvin C, Poupon R, de Lédinghen V, Beaugrand M. Non-invasive assessment of liver fibrosis by stiffness measurement in patients with chronic hepatitis B. Liver Int. 2009; 29: 242-247.

8. World Health Organization. Guidelines for the prevention, care and treatment of persons with chronic hepatitis B infection. Geneva: World Health Organization; 2015.

9. Kim DY, Kim SU, Ahn SH, Park JY, Lee JM, Park YN, Yoon KT, Paik YH, Lee KS, Chon CY, Han KH. Usefulness of FibroScan for detection of early compensated liver cirrhosis in chronic hepatitis B. Dig Dis Sci. 2009; 54: $1758-1763$.

10. Lin ZH, Xin YN, Dong QJ, Wang Q, Jiang XJ, Zhan SH, Sun Y, Xuan SY. Performance of the aspartate aminotransferase-to-platelet ratio index for the staging of hepatitis C-related fibrosis: an updated meta-analysis. Hepatology. 2011; 53: 726-736.

11. Wai CT, Greenson JK, Fontana RJ, Kalbfleisch JD, Marrero JA, Conjeevaram HS, Lok AS. A simple noninvasive index can predict both significant fibrosis and cirrhosis in patients with chronic hepatitis C. Hepatology. 2003; 38: 518-526.

12. Imbert-Bismut F, Ratziu V, Pieroni L, Charlotte F, Benhamou Y, Poynard T, MULTIVIRC Group. Biochemical markers of liver fibrosis in patients with hepatitis $\mathrm{C}$ virus infection: a prospective study. Lancet. 2001; 357: 1069-1075.

13. Vallet-Pichard A, Mallet V, Pol S. FIB-4: a simple, inexpensive and accurate marker of fibrosis in HCVinfected patients. Hepatology. 2006; 44: 769-770.

14. Xiao G, Yang J, Yan L. Comparison of diagnostic accuracy of aspartate aminotransferase to platelet ratio index and fibrosis-4 index for detecting liver fibrosis in adult patients with chronic hepatitis B virus infection: a systemic review and meta-analysis. Hepatology. 2015; 61: 292-302.

15. Kim WR, Berg T, Asselah T, Flisiak R, Fung S, Gordon SC, Janssen HL, Lampertico P, Lau D, Bornstein JD, Schall RE, Dinh P, Yee LJ, et al. Evaluation of APRI and FIB-4 scoring systems for non-invasive assessment of hepatic fibrosis in chronic hepatitis B patients. J Hepatol. 2016; 64: 773-780.

16. Lemoine M, Shimakawa Y, Nayagam S, Khalil M, Suso P, Lloyd J, Goldin R, Njai HF, Ndow G, Taal M, Cooke G, D’Alessandro U, Vray M, et al. The gamma-glutamyl transpeptidase to platelet ratio (GPR) predicts significant liver fibrosis and cirrhosis in patients with chronic HBV infection in West Africa. Gut. 2016; 65: 1369-1376.

17. Li Q, Song J, Huang Y, Li X, Zhuo Q, Li W, Chen C, Lu C, Qi X, Chen L. The gamma-glutamyl-transpeptidase to platelet ratio does not show advantages than APRI and Fib-4 in diagnosing significant fibrosis and cirrhosis in patients with chronic hepatitis B: a retrospective cohort study in China. Medicine (Baltimore). 2016; 95: e3372.

18. Schiavon LL, Narciso-Schiavon JL, Ferraz ML, Silva AE, Carvalho-Filho RJ. The $\gamma$-glutamyl transpeptidase to platelet ratio (GPR) in HBV patients: just adding up? Gut. 2017; 66: 1169-1170.

19. Branchi F, Conti CB, Baccarin A, Lampertico P, Conte D, Fraquelli M. Non-invasive assessment of liver fibrosis in chronic hepatitis B. World J Gastroenterol. 2014; 20: 14568-14580.

20. Enomoto M, Morikawa H, Tamori A, Kawada N. Noninvasive assessment of liver fibrosis in patients with chronic hepatitis B. World J Gastroenterol. 2014; 20: 12031-12038.

21. Cheng J, Hou J, Ding H, Chen G, Xie Q, Wang Y, Zeng M, $\mathrm{Ou} \mathrm{X}, \mathrm{Ma} \mathrm{H}$, Jia J. Validation of ten noninvasive diagnostic models for prediction of liver fibrosis in patients with chronic hepatitis B. PLoS One. 2015; 10: e0144425.

22. Bedossa P, Poynard T. An algorithm for the grading of activity in chronic hepatitis C. The METAVIR Cooperative Study Group. Hepatology. 1996; 24: 289-293.

23. Sterling RK, Lissen E, Clumeck N, Sola R, Correa MC, Montaner J, S Sulkowski M, Torriani FJ, Dieterich DT, Thomas DL, Messinger D, Nelson M, APRICOT Clinical Investigators. Development of a simple noninvasive index to predict significant fibrosis in patients with $\mathrm{HIV} / \mathrm{HCV}$ coinfection. Hepatology. 2006; 43: 1317-1325.

24. Giannini E, Risso D, Botta F, Chiarbonello B, Fasoli A, Malfatti F, Romagnoli P, Testa E, Ceppa P, Testa R. Validity 
and clinical utility of the aspartate aminotransferase-alanine aminotransferase ratio in assessing disease severity and prognosis in patients with hepatitis $\mathrm{C}$ virus-related chronic liver disease. Arch Intern Med. 2003; 163: 218-224.

25. Alkhouri N, Morris-Stiff G, Campbell C, Lopez R, Tamimi TA, Yerian L, Zein NN, Feldstein AE. Neutrophil to lymphocyte ratio: a new marker for predicting steatohepatitis and fibrosis in patients with nonalcoholic fatty liver disease. Liver Int. 2012; 32: 297-302.

26. Castera L. Noninvasive methods to assess liver disease in patients with hepatitis B or C. Gastroenterology. 2012; 142: 1293-1302.e4.

27. Eminler AT, Ayyildiz T, Irak K, Kiyici M, Gurel S, Dolar E, Gulten M, Nak SG. AST/ALT ratio is not useful in predicting the degree of fibrosis in chronic viral hepatitis patients. Eur J Gastroenterol Hepatol. 2015; 27: 1361-1366.

28. Chen B, Ye B, Zhang J, Ying L, Chen Y. RDW to platelet ratio: a novel noninvasive index for predicting hepatic fibrosis and cirrhosis in chronic hepatitis B. PLoS One. 2013; 8: e68780.

29. Ekiz F, Yüksel O, Koçak E, Yılmaz B, Altınbaş A, Çoban S, Yüksel I, Üsküdar O, Köklü S. Mean platelet volume as a fibrosis marker in patients with chronic hepatitis B. J Clin Lab Anal. 2011; 25: 162-165.

30. Hui AY, Chan HL, Wong VW, Liew CT, Chim AM, Chan FK, Sung JJ. Identification of chronic hepatitis B patients without significant liver fibrosis by a simple noninvasive predictive model. Am J Gastroenterol. 2005; 100: 616-623.

31. Zeng MD, Lu LG, Mao YM, Qiu DK, Li JQ, Wan MB, Chen CW, Wang JY, Cai X, Gao CF, Zhou XQ. Prediction of significant fibrosis in $\mathrm{HBeAg}$-positive patients with chronic hepatitis B by a noninvasive model. Hepatology. 2005; 42: 1437-1445.

32. Pan JJ, Yang CF, Chu CJ, Chang FY, Lee SD. Prediction of liver fibrosis in patients with chronic hepatitis B by serum markers. Hepatogastroenterology. 2007; 54: 1503-1506.

33. Aster RH. Pooling of platelets in the spleen: role in the pathogenesis of "hypersplenic" thrombocytopenia. J Clin Invest. 1966; 45: 645-657.

34. Kawasaki T, Takeshita A, Souda K, Kobayashi Y, Kikuyama M, Suzuki F, Kageyama F, Sasada Y, Shimizu E, Murohisa G, Koide S, Yoshimi T, Nakamura H, et al. Serum thrombopoietin levels in patients with chronic hepatitis and liver cirrhosis. Am J Gastroenterol. 1999; 94: 1918-1922.

35. Felker GM, Allen LA, Pocock SJ, Shaw LK, McMurray JJ, Pfeffer MA, Swedberg K, Wang D, Yusuf S, Michelson EL, Granger CB, CHARM Investigators. Red cell distribution width as a novel prognostic marker in heart failure: data from the CHARM Program and the Duke Databank. J Am Coll Cardiol. 2007; 50: 40-47.

36. Oh J, Kang SM, Hong N, Choi JW, Lee SH, Park S, Shin MJ, Jang Y, Chung N. Relation between red cell distribution width with echocardiographic parameters in patients with acute heart failure. J Card Fail. 2009; 15: 517-522.

37. Hampole CV, Mehrotra AK, Thenappan T, GombergMaitland M, Shah SJ. Usefulness of red cell distribution width as a prognostic marker in pulmonary hypertension. Am J Cardiol. 2009; 104: 868-872.

38. Lee HW, Kang W, Kim BK, Kim SU, Park JY, Kim DY, Ahn SH, Park YN, Han KH. Red cell volume distribution width-to-platelet ratio in assessment of liver fibrosis in patients with chronic hepatitis B. Liver Int. 2016; 36: 24-30.

39. Walters JG, Garrity PF. RDW-SD and RDW-CV: their relationship to RBC distribution curves and anisocytosis. Sysmex J Int. 1993; 3: 40-45.

40. Caporal FA, Comar SR. Evaluation of RDW-CV, RDW-SD, and MATH-1SD for the detection of erythrocyte anisocytosis observed by optical microscopy. J Bras Patol E Med Lab. 2013; 49: 324-331.

41. Schmilovitz-Weiss H, Tovar A, Halpern M, Sulkes J, Braun M, Rotman Y, Tur-Kaspa R, Ben-Ari Z. Predictive value of serum globulin levels for the extent of hepatic fibrosis in patients with chronic hepatitis B infection. J Viral Hepat. 2006; 13: 671-677.

42. Liu XD, Wu JL, Liang J, Zhang T, Sheng QS. Globulinplatelet model predicts minimal fibrosis and cirrhosis in chronic hepatitis B virus infected patients. World J Gastroenterol. 2012; 18: 2784-2792.

43. Yu MC, Chan KM, Lee CF, Lee YS, Eldeen FZ, Chou HS, Lee WC, Chen MF. Alkaline phosphatase: does it have a role in predicting hepatocellular carcinoma recurrence? J Gastrointest Surg. 2011; 15: 1440-1449.

44. Seto WK, Lee CF, Lai CL, Ip PP, Fong DY, Fung J, Wong DK, Yuen MF. A new model using routinely available clinical parameters to predict significant liver fibrosis in chronic hepatitis B. PLoS One. 2011; 6: e23077.

45. Lin CL, Liu CH, Wang CC, Liang CC, Su TH, Liu CJ, Kao JH. Serum biomarkers predictive of significant fibrosis and cirrhosis in chronic hepatitis B. J Clin Gastroenterol. 2015; 49: 705-713.

46. Chan HL, Wong ML, Hui AY, Hung LC, Chan FK, Sung JJ. Hepatitis B virus genotype $\mathrm{C}$ takes a more aggressive disease course than hepatitis B virus genotype B in hepatitis B e antigen-positive patients. J Clin Microbiol. 2003; 41: 1277-1279. 